\title{
Early outcomes after intrathecal baclofen therapy in ambulatory patients with multiple sclerosis
}

\author{
Bryan S. Lee, MD,, Jaes Jones, BS, ${ }^{2}$ Min Lang, MS, ${ }^{2}$ Rebecca Achey, BS, ${ }^{2}$ Lu Dai, MS, ${ }^{2}$ \\ Darlene A. Lobel, MD, ${ }^{1,4}$ Sean J. Nagel, MD, ${ }^{1,4}$ Andre G. Machado, MD, PhD, ${ }^{1,4}$ and \\ Francois Bethoux, MD 4,5 \\ ${ }^{1}$ Department of Neurosurgery, Neurological Institute, Cleveland Clinic; ${ }^{2}$ Cleveland Clinic Lerner College of Medicine, Case \\ Western Reserve University; ${ }^{3}$ School of Medicine, Case Western Reserve University; ${ }^{4}$ Center for Neurological Restoration, \\ Neurological Institute, Cleveland Clinic; and 5 Mellen Center for Multiple Sclerosis Treatment and Research, Neurological \\ Institute, Cleveland Clinic, Cleveland, Ohio
}

\begin{abstract}
OBJECTIVE Multiple sclerosis (MS) is a chronic autoimmune disease that causes demyelination and axonal loss. Walking difficulties are a common and debilitating symptom of MS; they are usually caused by spastic paresis of the lower extremities. Although intrathecal baclofen (ITB) therapy has been reported to be an effective treatment for spasticity in MS, there is limited published evidence regarding its effects on ambulation. The goal of this study was to characterize ITB therapy outcomes in ambulatory patients with MS.
\end{abstract}

METHODS Data from 47 ambulatory patients with MS who received ITB therapy were analyzed retrospectively. Outcome measures included Modified Ashworth Scale, Spasm Frequency Scale, Numeric Pain Rating Scale, and the Timed 25-Foot Walk. Repeated-measures ANOVA was used to test for changes in outcome measures between baseline and posttreatment ( 6 months and 1 year). Significance was set at $p<0.05$. Descriptive data are expressed as the mean \pm $\mathrm{SD}$, and results of the repeated-measures ANOVA tests and the Wilcoxon rank-sum test are expressed as the mean \pm SEM.

RESULTS There was a statistically significant reduction in the following variables: 1) aggregate lower-extremity Modified Ashworth Scale scores (from $14.8 \pm 1.0$ before ITB therapy to $5.8 \pm 0.8$ at 6 months posttreatment and $6.4 \pm 0.9$ at 1 year [p < 0.05]); 2) Numeric Pain Rating Scale scores ( $4.4 \pm 0.5$ before ITB, $2.8 \pm 0.5$ at 6 months, and $2.4 \pm 0.4$ at 1 year [ $p<0.05]) ; 3$ ) spasm frequency ( $45.7 \%$ of the patients reported a spasm frequency of $\geq 1$ event per hour before ITB therapy, whereas $15.6 \%$ and $4.3 \%$ of the patients reported the same at 6 months and 1 year posttreatment, respectively $[p<0.05]$ ); and 4$)$ the number of oral medications taken for spasticity $(p<0.05)$. Of the 47 patients, 34 remained ambulatory at 6 months, and 32 at 1 year posttreatment. There was no statistically significant change in performance on the Timed 25-Foot Walk test over time for those patients who remained ambulatory.

CONCLUSIONS In this retrospective study, the authors found that ITB therapy is effective in reducing spasticity and related symptoms in ambulatory patients with MS. Because the use of ITB therapy is increasing in ambulatory patients with MS, randomized, prospective studies are important to help provide a more useful characterization of the effects of ITB therapy on ambulation.

https://thejns.org/doi/abs/10.3171/2017.5.JNS162925

KEY WORDS intrathecal baclofen; multiple sclerosis; spasticity; ambulation; functional neurosurgery

$\mathrm{M}$ ULTIPLE sclerosis (MS) is a chronic, autoimmune disease characterized by the demyelination of the CNS and eventual axonal loss. This leads to both physical and cognitive disabilities in patients diagnosed with this disease. ${ }^{7}$ It is estimated that 400,000 individuals are affected by MS in the US alone, and up to $75 \%$ of these individuals have difficulty with ambulation. ${ }^{22}$ In a survey of more than 1000 persons with MS and 317 of their caregivers, the prevalence of walking difficulty was reported to be $41 \%$. In addition, fully $70 \%$ of these individuals described this as their most challenging symptom. ${ }^{14}$ Gait disturbance is often multifactorial in patients with MS. Common sources of neurological dysfunction, including weakness, spasticity, ataxia, sensory loss, visual deficits,

ABBREVIATIONS ITB = intrathecal baclofen; MAS = Modified Ashworth Scale; MS = multiple sclerosis; NARCOMS = North American Research Committee on MS; NPRS = Numeric Pain Rating Scale; SFS = Spasm Frequency Scale; T25FW = Timed 25-Foot Walk.

SUBMITTED November 23, 2016. ACCEPTED May 30, 2017.

INCLUDE WHEN CITING Published online December 1, 2017; DOI: 10.3171/2017.5.JNS162925. 
cognitive impairment, fatigue, and neuropathic pain, accumulate and may be magnified by relatively common comorbidities that are often acquired with age (cardiovascular disease, obesity, and joint deterioration). ${ }^{5}$

Spasticity is a velocity-dependent increase in stretch reflexes that is thought to be related to an imbalance in the excitatory and inhibitory signals that regulate motor neurons. ${ }^{14}$ Spasticity manifests clinically as muscle stiffness, spasms, clonus, and difficulty with voluntary movement due to co-contraction of agonist and antagonist muscles. In particular, spasticity has an outsized role in preventing normal ambulation. ${ }^{18}$ Rizzo et al. conducted a survey of more than 20,000 patients in the North American Research Committee on MS (NARCOMS) registry, and reported that $84 \%$ of responders had symptomatic spasticity and $34 \%$ had moderate to severe spasticity. ${ }^{20}$

A variety of treatments are available for the management of spasticity in MS. ${ }^{12}$ Baclofen is a $\gamma$-aminobutyric acid-B $\left(\mathrm{GABA}_{\mathrm{B}}\right)$ agonist with demonstrated efficacy on spasticity. ${ }^{10}$ Baclofen can be administered orally or intrathecally. Intrathecal baclofen (ITB) therapy is an FDA-approved treatment for spasticity of cerebral or spinal origin that is refractory to oral medications. Long-term ITB therapy requires the implantation of a programmable pump that delivers medication through a tunneled intrathecal catheter. Intrathecal baclofen therapy has been shown to be effective in reducing spasticity in MS and other CNS disorders. ${ }^{4}$ In the NARCOMS survey cited above, patients suffering from spasticity treated with ITB therapy reported significantly higher satisfaction with spasticity control than patients whose spasticity was managed with oral medications..$^{20}$ The risks and potential complications of ITB therapy are well known, and must be weighed against the potential benefits at the time of the treatment decision. ${ }^{1}$

Of note, most studies of ITB therapy in patients with MS were limited to nonambulatory patients, and quantitative monitoring of walking performance was generally not reported despite the fact that the use of ITB therapy is growing in ambulatory patients. ${ }^{5}$ However, the potential functional gain from spasticity control derived from ITB therapy may be offset by decreased strength output and a net negative effect on ambulation. The goal of our study was to characterize the outcomes of ITB therapy in a group of ambulatory patients with MS, including walking performance.

\section{Methods}

From an institutional review board-approved ITB therapy registry, we identified patients with a documented diagnosis of MS established by a neurologist, who underwent implantation of an intrathecal infusion system ("baclofen pump") after a successful test injection at our institution. Only patients who were able to perform the Timed 25-Foot Walk (T25FW) test with or without an assistive device before surgery, and for whom clinical data were available at baseline prior to surgery, at 6 months, and at 1 year after surgery, were included. Data extracted from the patients' electronic medical record included the following: 1) baseline demographic and disease characteristics (age, sex, symptom duration), and 2) outcome data at baseline, followed by 2 routine follow-up visits at 6 months $( \pm 3$ months) and 1 year ( \pm 3 months) after ITB therapy initiation. Outcome measures included the following: the Modified Ashworth Scale (MAS), the Spasm Frequency Scale (SFS), the Numeric Pain Rating Scale (NPRS), ambulation status, the $\mathrm{T} 25 \mathrm{FW}$, daily dose requirements for oral baclofen and tizanidine medications, and total number of symptomatic medications prescribed for spasticity.

\section{Modified Ashworth Scale}

The MAS is derived from the original Ashworth scale, first described in 1964, and is designed to assess muscle group resistance to passive mobilization on a 5-point ordinal scale. ${ }^{17}$ Bohannon and Smith added an intermediate grade to increase the sensitivity of the test, thereby implementing the 6-point ordinal MAS, which has become the most widely used spasticity scale in clinical trials of interventions for spasticity. ${ }^{8}$ The MAS scores were assessed in bilateral lower-extremity muscle groups, including the hip adductors, knee flexors, knee extensors, and ankle plantar flexors. Scores for all muscle groups were summed to obtain a global lower-extremity MAS spasticity score. For all patients included in this study, the MAS was clinically collected by 1 of 2 board-certified physiatrists. Of note, only 5 of the 47 patients included in the study received physical therapy during the year following ITB initiation.

\section{Spasm Frequency Scale}

The SFS is an ordinal measure of spasm frequency with a 5-point score range: 0 (no spasms), 1 (spasms with stimulation), 2 (occasional spasms), 3 (more than 1 spasm per hour but fewer than 10), and 4 (10 or more spasms per hour). ${ }^{19}$ This scale has been shown to be sensitive to the effect of ITB therapy on spasticity. ${ }^{5}$

\section{Numeric Pain Rating Scale}

The NPRS is widely used. ${ }^{26}$ Patients were asked to rate their level of leg pain in the past 24 hours between 0 and 10 , with 0 signifying no pain and 10 signifying the worst pain.

\section{The Timed 25-Foot Walk}

The T25FW is a component of the Multiple Sclerosis Functional Composite, a 3-part, quantitative assessment tool aimed at measuring outcomes for MS-focused clinical trials. ${ }^{11,21,25}$ The T25FW is a well-validated test to measure the speed of ambulation over a short distance in patients with MS. The patient is instructed to walk 25 feet as quickly and safely as possible. The use of assistive devices is permitted. For this study, data were collected on $\mathrm{T} 25 \mathrm{FW}$ results and type of assistive device required to complete the test.

\section{Statistical Analysis}

Descriptive statistics were reported as the mean \pm SD. Repeated-measures ANOVA tests were performed for statistical comparison between baseline, 6 months after, and 1 year after ITB therapy initiation. Post hoc analysis of pairwise comparisons was performed using the Bonfer- 
roni method. Results of the repeated-measures ANOVA tests were reported as the mean \pm SEM. Fisher's exact tests were run for statistical comparison of the proportion of patients reporting more than 1 spasm per hour at baseline, at 6 months, and at 1 year post-ITB therapy. A Wilcoxon rank-sum test was used to compare baseline T25FW results between patients who remained ambulatory at 1 year and those who lost the ability to ambulate. Results of the Wilcoxon rank-sum test were reported as the mean \pm SEM. Significance for all statistical analyses was set at $\mathrm{p}<0.05$.

\section{Results}

\section{Descriptive Statistics}

Forty-seven ambulatory patients who underwent ITB pump implantation surgery between 2001 and 2013 were included in the analyses. The demographic and disease characteristics of the patients are presented in Table 1.

\section{Effects of ITB Therapy on Aggregate MAS Score, NPRS Score, and SFS Score}

Forty-three of 47 patients completed MAS scores at all time points and were used for analysis. We included the total subset of patients who were ambulatory at baseline, and then reported on the subsets of patients who had complete data for each specific outcome assessment. The aggregate MAS score for lower-extremity muscles was significantly reduced, from $14.8 \pm 1.0$ before ITB therapy to $5.7 \pm 0.8$ at 6 months post-ITB $(\mathrm{p}<0.0001)$ and $6.0 \pm 0.8$ at 1 year post-ITB $(\mathrm{p}<0.0001)$ (overall repeated-measures ANOVA significance, $\mathrm{p}<0.0001$ ) (Fig. 1). Thirty-eight patients with complete data for all 3 times points were included for analysis of pain severity. Pain scores were significantly reduced, from $4.4 \pm 0.6$ before ITB therapy to $2.8 \pm 0.5$ at 6 months post-ITB $(\mathrm{p}<0.05)$ and $2.4 \pm 0.5$ at 1 year post-ITB $(\mathrm{p}<0.005)$ (overall repeated-measures ANOVA significance, $p=0.0028$ ) (Fig. 2). For spasm frequency, Fisher's exact tests were used to compare the proportion of patients at each time point. Prior to ITB ther-

TABLE 1. Summary statistics for 47 ambulatory patients before initiation of ITB therapy

\begin{tabular}{cc}
\hline \multicolumn{1}{c}{ Factor } & Summary \\
\hline Age & $58.2 \pm 8.6$ \\
\hline Sex & $16(34)$ \\
\hline Male & $31(66)$ \\
\hline Female & $16.1 \pm 8.6$ \\
\hline Disease duration (yrs) & $11(23.4)$ \\
\hline MS type & $5(10.6)$ \\
\hline Relapsing-remitting & $2(4.3)$ \\
\hline Primary progressive & $26(55.3)$ \\
\hline Progressive relapsing & $3(6.4)$ \\
\hline Secondary progressive &
\end{tabular}

Continuous data are presented as the mean $\pm \mathrm{SD}$. Categorical data are presented as the number (\%).

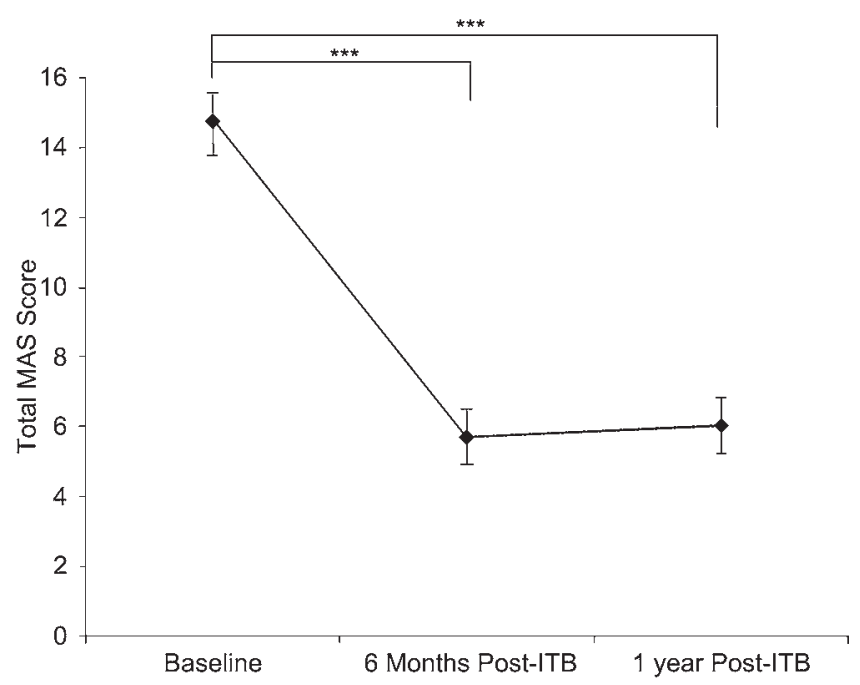

FIG. 1. Graph showing MAS score compared between baseline, 6 months post-ITB, and 1 year post-ITB. ${ }^{* * *} p<0.0001$.

apy, $45.7 \%$ of the patients reported a spasm frequency of $\geq 1$ event per hour, and $15.6 \%$ and $4.3 \%$ of the patients reported the same at 6 months and 1 year after ITB therapy initiation, respectively. The proportion of patients reporting spasm frequencies more than once per hour was significantly reduced from baseline to 6 months after initiation of ITB therapy, and from baseline to 1 year after ITB therapy initiation ( $p=0.0029$ and $p<0.0001$, respectively). The proportion of patients reporting spasms more than once per hour was not significantly reduced from 6 months to 1 year post-ITB therapy $(p=0.087)($ Fig. $3 \mathrm{~A})$. Furthermore, spasm frequency was significantly improved both in groups of patients who remained ambulatory at 1-year follow-up and in those who were nonambulatory at 1-year follow-up, suggesting that spasticity was controlled without compromising ambulation (Fig. 3B and C).

\section{Ambulatory Status and Walking Speed Following ITB Therapy Initiation}

Of the 47 patients who were ambulatory prior to ITB therapy initiation, $34(72.3 \%)$ patients remained ambulatory at 6 months post-ITB; $32(68.1 \%)$ patients remained ambulatory at 1 year post-ITB. Those who remained am-

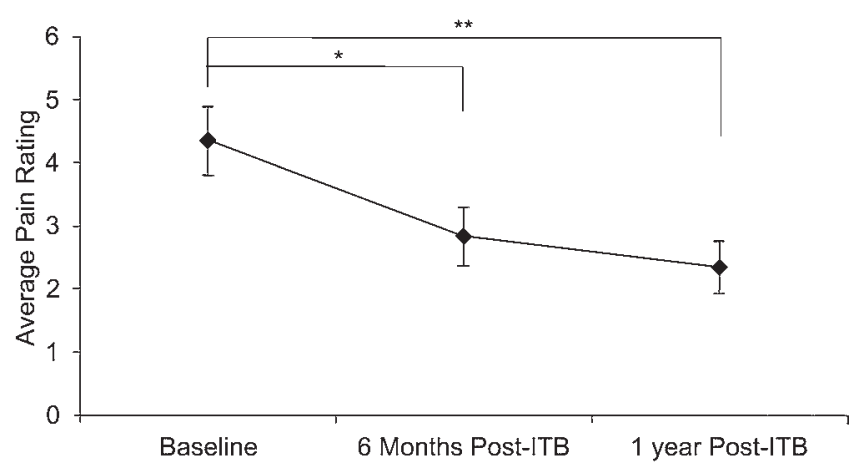

FIG. 2. Graph showing NPRS score compared between baseline, 6 months post-ITB, and 1 year post-ITB. ${ }^{*} p<0.05,{ }^{* *} p<0.005$. 

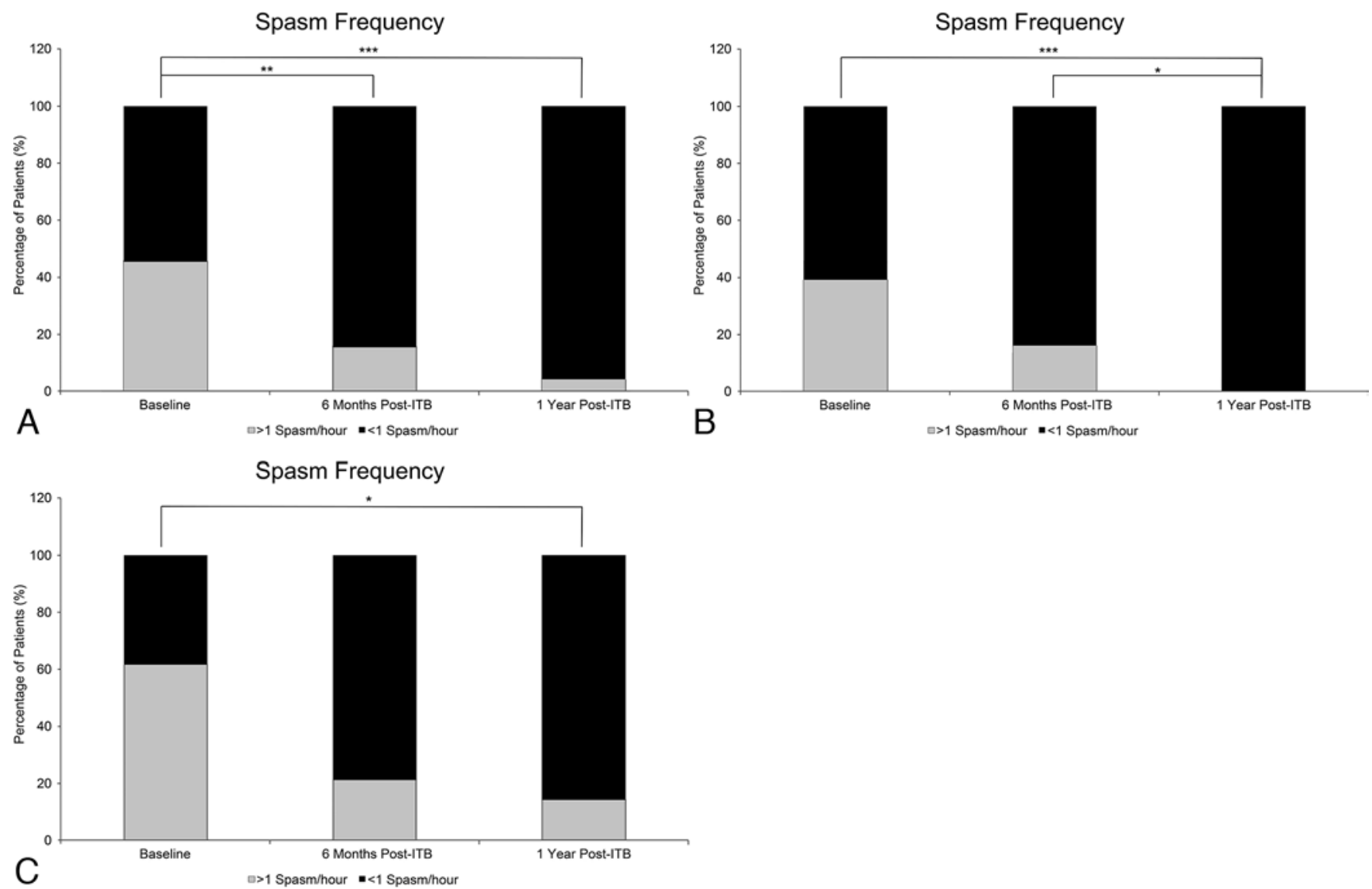

FIG. 3. Bar graphs showing outcomes after ITB therapy. A: Spasm frequency compared between baseline, 6 months post-ITB, and 1 year post-ITB in both ambulatory and nonambulatory groups. ${ }^{* *} p<0.005,{ }^{* * *} p<0.0001$. B: Spasm frequency compared between baseline, 6 months post-ITB, and 1 year post-ITB in the ambulatory group. ${ }^{*} p<0.05,{ }^{* * *} p<0.0001$. C: Spasm frequency compared between baseline, 6 months post-ITB, and 1 year post-ITB in the nonambulatory group. ${ }^{\star} p<0.05$.

bulatory at 1 year had walked faster on the T25FW at baseline than those who lost the ability to ambulate $(22.1$ \pm 3.4 seconds vs $62 \pm 11.1$ seconds, $\mathrm{p}<0.0001$ ) (Fig. 4). Twenty-seven of the 32 patients who were ambulatory at 1 year post-ITB had completed T25FW tests for all 3 time points. Of these patients, the average time to complete the T25FW was $33.8 \pm 6.1$ seconds at baseline, $22.8 \pm 3.1 \mathrm{sec}-$ onds at 6 months post-ITB, and $27.7 \pm 4.3$ seconds at 1 year post-ITB. There were no statistically significant differences between baseline and follow-up visits $(\mathrm{p}=0.28)$ (Fig. 5). The daily dose of ITB was $146.5 \pm 117.4 \mu \mathrm{g} /$ day at 6 months post-ITB and $200.6 \pm 185.5 \mu \mathrm{g} /$ day at 1 year postITB. Daily dose of ITB did not correlate with a change in T25FW results at 6 months post-ITB $(\mathrm{p}=0.26)$ or at 1 year post-ITB $(p=0.47)$. In addition, physical therapy did not correlate with walking status at 6 months post-ITB ( $p$ $>0.95)$ or at 1 year post-ITB $(p=0.78)$. Furthermore, there was no statistically significant difference between type of assistive devices used at baseline, 6 months post-ITB, or 1 year post-ITB $(\mathrm{p}=0.85)$.

\section{Reduction in Daily Dose of Oral Baclofen and Tizanidine Following Initiation of ITB Therapy}

The number of medications used for spasticity was found to be decreased 6 months and 1 year following ini- tiation of ITB therapy $(p=0.0001)$ (Table 2). Completed data quantifying the daily dose of oral baclofen and tizanidine at all 3 time points were included for 17 and 16 patients, respectively. Prior to ITB therapy initiation, oral baclofen and tizanidine daily dose requirements were 75.6 $\pm 4.8 \mathrm{mg} / \mathrm{day}$ and $6.6 \pm 2.2 \mathrm{mg} / \mathrm{day}$, respectively. Compared with baseline, oral baclofen and tizanidine daily dose requirements were significantly reduced at 6 months after ITB therapy initiation $(31.8 \pm 8.6 \mathrm{mg} / \mathrm{day}[\mathrm{p}=0.0001]$ and $2.25 \pm 1.3 \mathrm{mg} /$ day $[\mathrm{p}=0.0203]$, respectively). Oral baclofen dose was reduced at 1 year after ITB therapy initiation $(36.2 \pm 9.4 \mathrm{mg} / \mathrm{day}[\mathrm{p}=0.0009])$. However, there was no statistically significant difference in oral baclofen dose requirements between 6 months and 1 year after ITB therapy initiation $(p=0.99)$. Additionally, there was no statistically significant difference in tizanidine daily dose requirement between baseline and 1 year post-ITB $(2.5 \pm$ $1.5 \mathrm{mg} /$ day $[\mathrm{p}=0.124]$ ), or between 6 months and 1 year after ITB therapy initiation $(\mathrm{p}=0.99)$ (Fig. 6).

\section{Discussion}

The results from our retrospective analysis of a subgroup of ambulatory patients with MS who had severe spasticity indicate that ITB therapy can be effective in re- 


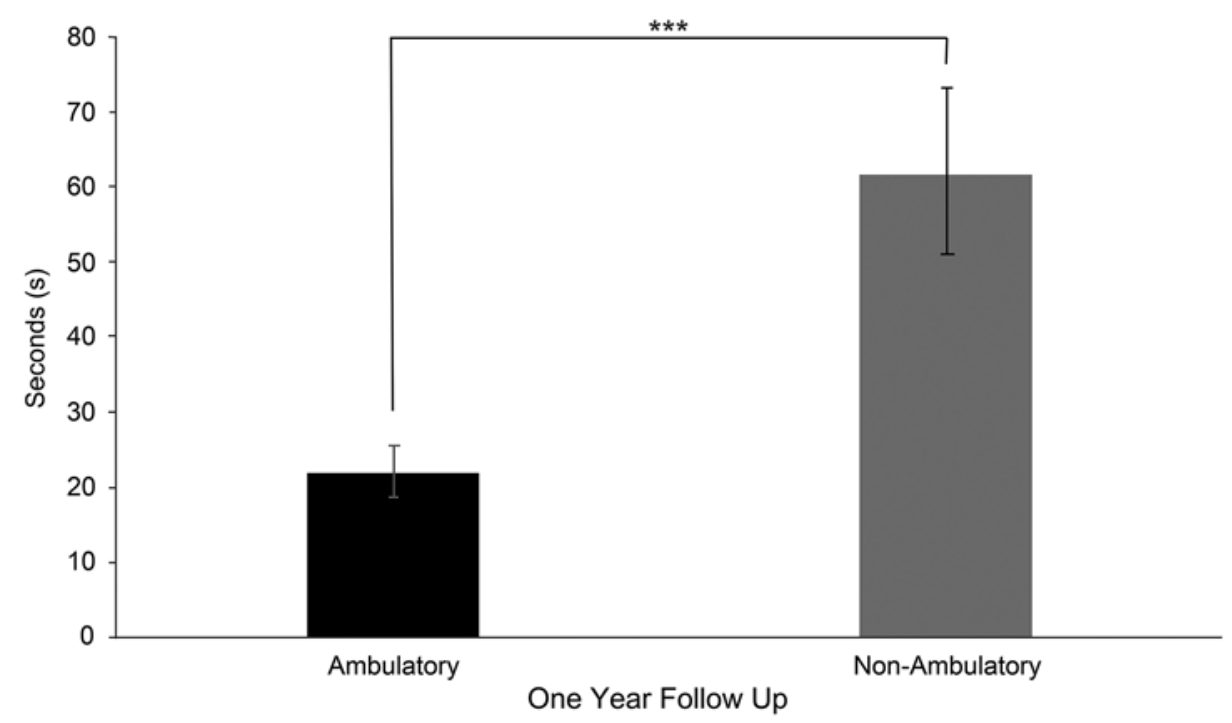

FIG. 4. Bar graph showing baseline T25FW test speed compared between patients who were ambulatory versus those who were nonambulatory at 1 -year follow-up. ${ }^{* *} p<0.0001$.

ducing spasticity and spasticity-related symptoms in select patients. Specifically, there was a significant reduction in aggregate MAS scores, NPRS scores, and the proportion of patients reporting more than 1 spasm per hour, from baseline to 6 months and 1 year after ITB therapy initiation.

Intrathecal baclofen for widespread use was first made available in the early 1990s to treat refractory lower-extremity spasticity in patients with MS. ${ }^{1}$ Historically, ITB was targeted at the nonambulatory population, where it was shown to provide effective symptomatic relief for spasticity-related pain, ease of care, ability to transfer, and posture. ${ }^{22}$ Reduced spasticity and increased treatment satisfaction were reported in a group of patients with MS who were receiving ITB compared with the controls using oral medications in a survey study. ${ }^{20}$ In a 5 -year prospective study of 21 patients with spasticity, including 53\% whose spasticity was related to MS, improved clinical efficacy of spasticity control measured using the MAS and higher patient satisfaction were noted in those who received ITB therapy. However, absent was a significant improvement of global disability and subjective health status measures, including the expanded disability status scale, sickness im-

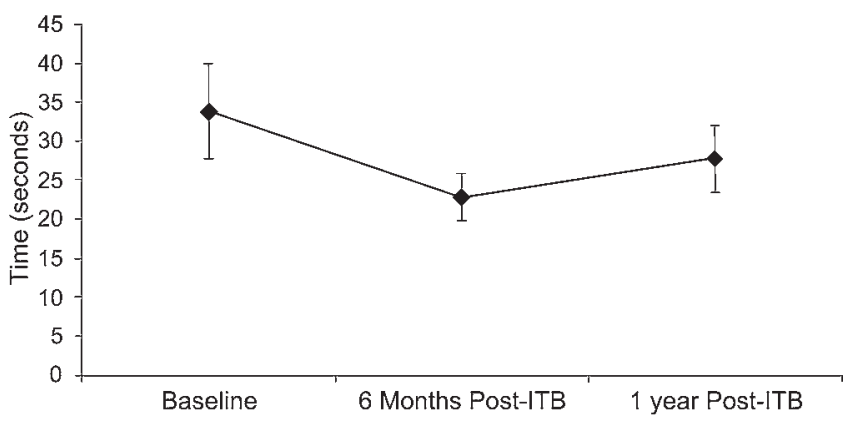

FIG. 5. Graph showing T25FW test results compared between baseline, 6 months post-ITB, and 1 year post-ITB. pact profile, and Hopkins checklist system..$^{27}$ Intrathecal baclofen is now routinely offered to ambulatory patients with MS. This group has the potential for improved global disability because it is expected that they should be able to participate in a wider range of activities. ${ }^{6,22}$

Ambulatory patients with MS are at risk for loss of function from increased lower-extremity weakness following ITB use. Although the majority of patients retained the ability to walk following ITB therapy in our study, a subset of patients (approximately 30\%) lost the ability to ambulate following this therapy. Although it is not possible to definitively determine the cause of the loss of ambulation, we speculate that the natural progression of the disease plays an important role in this decline. Drug-related complications of ITB therapy are relatively uncommon. ${ }^{1-3}$ The doses administered to our patients were also tightly controlled to allow for satisfactory response while minimizing risks of adverse effects, including muscular hypotonia, and are lower than the average doses used in nonambulatory patients. In patients who remained ambulatory, there was no statistically significant difference in walking speed between baseline and follow-up. This suggests that satisfactory control of spasticity could be achieved while maintaining mobility in those patients. However,

TABLE 2. Spasticity medication usage comparing baseline number of medications with 6 months and 1 year after initiation of ITB therapy

\begin{tabular}{cccc}
\hline $\begin{array}{c}\text { No. of Spasticity } \\
\text { Medications }\end{array}$ & Baseline & $\begin{array}{c}6 \text { Mos } \\
\text { Post-ITB }\end{array}$ & $\begin{array}{c}1 \mathrm{Yr} \\
\text { Post-ITB }\end{array}$ \\
\hline None & $2(4.3)$ & $21(44.7)$ & $23(48.9)$ \\
\hline 1 & $23(48.9)$ & $17(36.2)$ & $17(36.2)$ \\
\hline 2 & $20(42.6)$ & $8(17.0)$ & $6(12.8)$ \\
\hline 3 & $2(4.3)$ & $1(2.1)$ & $1(2.1)$ \\
\hline
\end{tabular}

Data are presented as the number (\%). 


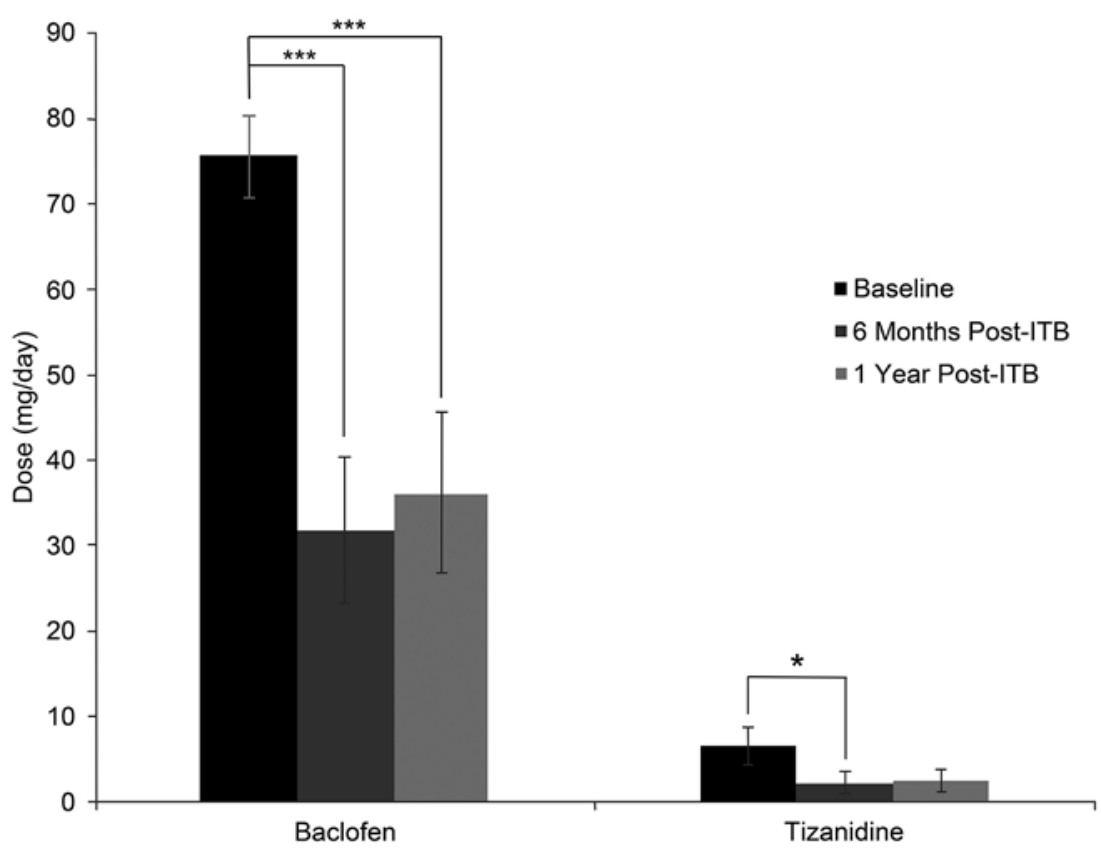

FIG. 6. Bar graph showing daily doses of oral antispastic agents compared between baseline, 6 months post-ITB, and 1 year postITB. ${ }^{*} p<0.05,{ }^{* * *} p<0.001$.

there appears to be a trend toward slower walking times 1 year following ITB therapy. The fact that patients who were nonambulatory at the end of the study walked more slowly on the $\mathrm{T} 25 \mathrm{FW}$ at baseline is not surprising, because slower gait is positively correlated with lower-extremity weakness. This finding does not provide information on the cause of the change in functional status, but could be useful in educating candidates for ITB therapy regarding potential functional outcomes at 6 months and 1 year. In our patients, an average baseline walking time of approximately 60 seconds was associated with loss of ambulation.

Oral antispasticity medications, including baclofen and tizanidine, are frequently used for symptom control in MS patients. These medications, because they are nonspecific when taken orally, exert systemic side effects, particularly sedation and weakness. Baclofen is also associated with dizziness and cognitive slowing, and potentially symptoms of withdrawal, which include tachycardia, hypertonia, hyperthermia, muscle rigidity, and hallucinations. ${ }^{15}$ Tizanidine, a central $\alpha_{2}$ adrenergic agonist, is commonly associated with nausea, emesis, dizziness, hallucinations, delirium, and depression..$^{13}$ In our study, there was a significant reduction in daily oral baclofen dose between baseline and 6 months or 1 year after ITB therapy initiation, whereas there was a significant reduction in tizanidine dose only from baseline to 6 months.

Surgery for ITB pump implantation is not without risks. Postoperative complications include infection (3\%-15\%), catheter obstruction or disconnection $(2 \%-16 \%)$, and CSF leakage (5\%-9\%). ${ }^{9,15}$ Catheter malfunction can be due to catheter fracture, dislocation, and granuloma formation at the tip of the catheter leading to occlusion, although with baclofen this remains debatable. Pump malfunction is associated with unexpected battery failure and motor lock. Malfunction of the pump or catheter precipitates baclofen withdrawal, which is manifested by pruritus, fever, hypertonia, tachycardia, and seizures. ${ }^{3,28}$ Baclofen overdose, which is less commonly associated with ITB system malfunction but is sometimes caused by human error, leads to sedation, hypotonia, cardiorespiratory depression, and coma. ${ }^{16}$ Both withdrawal and overdose are potentially lifethreatening conditions, although they are reversible with appropriate supportive care. There are significant up-front and recurring costs with ITB therapy, including the need for periodic refills and adjustments, and the need to undergo repeat surgery for battery replacement. There are several studies that show increased cost-effectiveness with ITB therapy. ${ }^{23,24}$

We recognize that there are several limitations in this study, most importantly that the data were collected retrospectively and were at times incomplete. Moreover, there is always a risk of introducing an unintended bias due to the nature of retrospective studies. We did not systematically administer quality of life measures, and therefore we did not report on the patients' perception of treatment outcomes. Our sample size was relatively small, and there was no comparison group of ambulatory patients with severe spasticity who were not treated with ITB. All patients who met the criteria were included, but there was no substratification within the ambulatory MS group based on the severity of MS. An enhanced evaluation of the effect of ITB on ambulation in MS patients is also needed to inform decisions about who would be the best candidates.

\section{Conclusions}

In this retrospective analysis of ambulatory patients diagnosed with MS, there was a significant reduction in spasticity, pain, spasms, and the daily dose of oral antispasticity medications with ITB therapy. Walking per- 
formance, assessed with the $\mathrm{T} 25 \mathrm{FW}$, remained stable in a majority of patients. This suggests that improvement in walking speed should not be expected with ITB therapy. However, adequate control of spasticity with preservation of ambulation is a reasonable expectation in a majority of patients. The loss of ambulation at 6 months and 1 year in patients with a slower walking time at baseline warrants additional study.

\section{References}

1. Abel NA, Smith RA: Intrathecal baclofen for treatment of intractable spinal spasticity. Arch Phys Med Rehabil 75:5458, 1994

2. Albright AL, Turner M, Pattisapu JV: Best-practice surgical techniques for intrathecal baclofen therapy. J Neurosurg 104 (4 Suppl):233-239, 2006

3. Alden TD, Lytle RA, Park TS, Noetzel MJ, Ojemann JG: Intrathecal baclofen withdrawal: a case report and review of the literature. Childs Nerv Syst 18:522-525, 2002

4. Azouvi P, Mane M, Thiebaut JB, Denys P, Remy-Neris O, Bussel B: Intrathecal baclofen administration for control of severe spinal spasticity: functional improvement and longterm follow-up. Arch Phys Med Rehabil 77:35-39, 1996

5. Bethoux F: Gait disorders in multiple sclerosis. Continuum (Minneap Minn) 19 (4 Multiple Sclerosis):1007-1022, 2013

6. Bethoux F, Gogol D, Schwetz K, Kinkel R: Use of a registry of intrathecal baclofen therapy in a large multiple sclerosis center: analysis of data on 82 patients and proposed changes. Arch Phys Med Rehabil 82:1329, 2001 (Abstract)

7. Bjartmar C, Wujek JR, Trapp BD: Axonal loss in the pathology of MS: consequences for understanding the progressive phase of the disease. J Neurol Sci 206:165-171, 2003

8. Bohannon RW, Smith MB: Interrater reliability of a modified Ashworth scale of muscle spasticity. Phys Ther 67:206-207, 1987

9. Borowski A, Littleton AG, Borkhuu B, Presedo A, Shah S, Dabney KW, et al: Complications of intrathecal baclofen pump therapy in pediatric patients. J Pediatr Orthop 30:7681,2010

10. Brar SP, Smith MB, Nelson LM, Franklin GM, Cobble ND: Evaluation of treatment protocols on minimal to moderate spasticity in multiple sclerosis. Arch Phys Med Rehabil 72:186-189, 1991

11. Fischer JS, Rudick RA, Cutter GR, Reingold SC: The multiple sclerosis functional composite measure (MSFC): an integrated approach to MS clinical outcome assessment. Mult Scler 5:244-250, 1999

12. Haselkorn JK, Balsdon Richer C, Fry Welch D, Herndon RM, Johnson B, Little JW, et al: Overview of spasticity management in multiple sclerosis. Evidence-based management strategies for spasticity treatment in multiple sclerosis. J Spinal Cord Med 28:167-199, 2005

13. Kamen L, Henney HR III, Runyan JD: A practical overview of tizanidine use for spasticity secondary to multiple sclerosis, stroke, and spinal cord injury. Curr Med Res Opin 24:425-439, 2008

14. Larocca NG: Impact of walking impairment in multiple sclerosis: perspectives of patients and care partners. Patient 4:189-201, 2011

15. Leo RJ, Baer D: Delirium associated with baclofen withdrawal: a review of common presentations and management strategies. Psychosomatics 46:503-507, 2005

16. Levitt MR, Browd SR: Spasticity: classification, diagnosis, and management, in Ellenbogen RG, Abdulrauf SL, Sekhar LN (eds): Principles of Neurological Surgery, ed 3. Philadelphia: Elsevier, 2012, pp 757-763
17. Pandyan AD, Johnson GR, Price CI, Curless RH, Barnes MP, Rodgers H: A review of the properties and limitations of the Ashworth and modified Ashworth Scales as measures of spasticity. Clin Rehabil 13:373-383, 1999

18. Pau M, Coghe G, Corona F, Marrosu MG, Cocco E: Effect of spasticity on kinematics of gait and muscular activation in people with multiple sclerosis. J Neurol Sci 358:339-344, 2015

19. Penn RD, Savoy SM, Corcos D, Latash M, Gottlieb G, Parke $\mathrm{B}$, et al: Intrathecal baclofen for severe spinal spasticity. $\mathbf{N}$ Engl J Med 320:1517-1521, 1989

20. Rizzo MA, Hadjimichael OC, Preiningerova J, Vollmer TL: Prevalence and treatment of spasticity reported by multiple sclerosis patients. Mult Scler 10:589-595, 2004

21. Rudick R, Antel J, Confavreux C, Cutter G, Ellison G, Fischer J, et al: Clinical outcomes assessment in multiple sclerosis. Ann Neurol 40:469-479, 1996

22. Sadiq SA, Wang GC: Long-term intrathecal baclofen therapy in ambulatory patients with spasticity. J Neurol 253:563569,2006

23. Saulino M, Guillemette S, Leier J, Hinnenthal J: Medical cost impact of intrathecal baclofen therapy for severe spasticity. Neuromodulation 18:141-149, 2015

24. Slof J, Serrano D, Álvarez M, Álvarez López-Dóriga M, Marqués T, Benito J, et al: Cost-effectiveness model results of intrathecal baclofen therapy compared to conventional medical management in patients with non-focal disabling spasticity who are resistant or intolerant to oral therapy at the Institut Guttmann. Value Health 17:A399-A400, 2014

25. Whitaker JN, McFarland HF, Rudge P, Reingold SC: Outcomes assessment in multiple sclerosis clinical trials: a critical analysis. Mult Scler 1:37-47, 1995

26. Williamson A, Hoggart B: Pain: a review of three commonly used pain rating scales. J Clin Nurs 14:798-804, 2005

27. Zahavi A, Geertzen JHB, Middel B, Staal M, Rietman JS: Long term effect (more than five years) of intrathecal baclofen on impairment, disability, and quality of life in patients with severe spasticity of spinal origin. J Neurol Neurosurg Psychiatry 75:1553-1557, 2004

28. Zuckerbraun NS, Ferson SS, Albright AL, Vogeley E: Intrathecal baclofen withdrawal: emergent recognition and management. Pediatr Emerg Care 20:759-764, 2004

\section{Disclosures}

Dr. Bethoux is a consultant for Merz and Ipsen. Dr. Machado is a patent holder with Ensure, ATI, and Cardionomics. He is also a consultant for St. Jude Hospital and Functional Neuromodulation.

\section{Author Contributions}

Conception and design: Lee, Lobel, Machado, Bethoux. Acquisition of data: Lee, Jones, Lang. Analysis and interpretation of data: Lee, Jones, Lang, Achey, Dai, Bethoux. Drafting the article: Lee, Jones, Lang. Critically revising the article: Lee, Lobel, Nagel, Bethoux. Reviewed submitted version of manuscript: Lee, Jones, Lang, Achey, Lobel, Nagel, Machado, Bethoux. Approved the final version of the manuscript on behalf of all authors: Lee. Statistical analysis: Lee, Jones, Lang, Achey. Administrative/techni$\mathrm{cal} /$ material support: Bethoux. Study supervision: Lobel, Nagel, Machado, Bethoux.

\section{Correspondence}

Bryan Lee, Department of Neurosurgery, Neurological Institute, Cleveland Clinic Foundation, 9500 Euclid Ave., S40, Cleveland, OH 44195. email: bryansukbae.lee@gmail.com. 